\title{
Clients' Perceptions of HIV/AIDS Supportive Counseling in Botswana: A Qualitative Study
}

\author{
Stockton $\mathrm{R}^{* 1}$, Paul $\mathrm{T}^{2}$, Mokalake $\mathrm{E}^{2}$, Morran $\mathrm{DK}^{3}$, Powless $\mathrm{MD}^{4}$, Goldberg $\mathrm{D}^{4}$, Li S${ }^{4}$, Blackwell N${ }^{4}$, and \\ Sharma $\mathbf{R}^{4}$
}

${ }^{1}$ Department of Counseling and Educational Psychology, Indiana University, Bloomington, USA

${ }^{2}$ Institute for Development Management, Botswana, Africa

${ }^{3}$ Indiana University-Purdue University Indianapolis, USA

${ }^{4}$ Indiana University, USA

${ }^{*}$ Corresponding author: Stockton R, Department of Counseling and Educational Psychology, Indiana University, Bloomington, IN 47405, E-mail: stocktor@indiana.edu

Citation: Stockton R, Paul T, Mokalake E, Morran DK, Powless MD, et al. (2017) Clients' Perceptions of HIV/AIDS Supportive Counseling in Botswana: A Qualitative Study. J Immunol Infect Dis 4(1): 102

Received Date: December 16, 2016 Accepted Date: June 13, 2017 Published Date: June 15, 2017

\begin{abstract}
The United Nations has created the Sustainable Development Goals (SDG) initiative in an attempt to end the HIV/AIDS epidemic worldwide by the year 2030. In an effort to assist in the SDG cause, the current study sought to obtain data from clients in Botswana regarding the perceptions of their experiences with HIV/AIDS counseling from 25 randomly selected hospitals/clinics. Clients were then sequentially offered the opportunity to participate in the study. Volunteers were then provided with the survey and data was collected through 5 survey questions that elicited qualitative feedback from participants. Once data was collected, thematic analysis was utilized to determine major themes found within participants' responses. The themes found within these responses are presented along with their implications for future research and practice.
\end{abstract}

Keywords: HIV/AIDS; Counseling; Botswana

\section{Introduction}

The United Nations has created the Sustainable Development Goals (SDGs) initiative that aims to ultimately end the AIDS epidemic worldwide by 2030 [1,2]. In this concerted effort to end AIDS, sub-Saharan Africa must be of primary focus as it represents the priority countries in eliminating child contraction of the HIV virus and keeping mothers alive [3] and due to the large numbers of individuals living with HIV/AIDS in these countries [3,4]. Among the many goals of the SDG initiatives is the aim to deliver mental health services to individuals living with HIV/AIDS [2]. With increased availability of ARV treatment [1] it is now possible for individuals to live with HIV as long as they adhere to medication protocols.

Although this route provides a means to live, contraction of HIV still results in living with a chronic disease. Individuals afflicted with HIV/AIDS suffer from psychological hardships such as emotional distress, uncertainty, helplessness, anxiety, depression, anger, thoughts of suicide, revenge, and negative social stigma perceived from others $[1,5]$. These psychological stressors may lead to impulsive or destructive behaviors (e.g., substance abuse) that interfere with medication adherence [6] and risky health behaviors that undermine treatment and contribute to the spread of HIVAIDS. In order to decrease the chances of psychological stressors leading to the spread of HIV/AIDS, counseling and mental health services are of primary importance in treating individuals afflicted with HIV/AIDS. Given the prevalence of HIV/AIDS in sub-Saharan Africa, the United Nations' SDG initiative, and the importance of mental health care in preventing the spread of HIV/AIDs, this article will explore the perceptions of HIV-positive clients about the counseling services provided to them in sub-Saharan Africa, specifically, Botswana.

\section{Counseling Services in Sub-Saharan Africa}

The majority of early research in this area has focused on counselors providing services. For example, in Zimbabwe counselors reported receiving minimal support and supervision as well as being undertrained [7]. Additionally, counselors indicated that the beginning and later stages of counseling were difficult (with the beginning stages being most difficult) and that working with serodiscordant couples (one partner HIV-positive, one HIV-negative) was problematic along with working with couples wanting to have children. In Kenya and Tanzania [8] counselors reported feeling pressured to be good role models in their communities and to provide information to their communities as they were perceived to be very knowledgeable. Moreover, counselors indicated that 
they felt work stress from both external contributors (e.g., economic conditions in their country), job structure contributors (e.g., heavy and unpredictable workload, compensation), and job duties (e.g., fear of clients). It is worth mentioning that although these counselors reported psychological stress due to their role as counselors, many of them found their work satisfying and gratifying $[7,8]$.

More recently there have been a number of studies relating to voluntary AIDS testing and counseling, the majority of the studies focus on the barriers in reaching out to the counseling and testing services, especially underserved communities. Typically there is a one-time counseling session after the voluntary test; however, particularly noteworthy is the idea of having mobile voluntary and counseling services (MVCT), an innovation that has proved quite successful in several countries $[9,10]$. The stigma associated with having HIV/AIDS and how counseling has proven to be helpful in this regard is also discussed by Mall, Middlekoop, Mark, Wood \& Becker (2013) [11].

A few studies of HIV-positive clients in Sub-Saharan Africa who participated in group counseling have also been reported. Schacham and colleagues [12] administered questionnaires to 397 clients in western Kenya who were HIV-positive and had self-enrolled in one or more psychosocial support groups. The questionnaire was designed to collect data related to participants' demographic and HIV-related characteristics and also included assessments of psychological distress. The authors reported that participants were primarily female (72\%), that 70\% were unemployed, and that participants had been living with HIV for an average of 2.5 years. They also reported that, across all participants, HIV status was rarely shared with sex partners, family members, or friends and that psychological distress was more prevalent among women on measures of depression, anxiety, paranoid ideation, interpersonal sensitivity, and somatization.

Edwards and Edwards [13] studied 30 HIV positive clients in South Africa who received six one-hour sessions of person centered group counseling. Compared to a student control group, the HIV positive clients showed improvements in perceptions of autonomy, personal growth, environmental mastery, positive relations with others, and self-acceptance. Specific to Botswana, Stockton, Paul, Morran, \& Mokalake (2016) [4] surveyed clients' perception of their experiences with counseling services and found quantitative data to provide evidence that a majority of HIV/AIDS clients reported a positive experience with counseling in Botswana. The current study goes into considerable depth regarding the counseling experienced by the participants taking into account not only the social stigma but multiple other aspects that counseling sessions focus on, such as medication adherence, safe sex practices, alcohol and drug issues and knowledge about HIV/AIDS, self-acceptance, disclosure to family, fear of future and many others. One important component of the current study is preventing mother to child transmission (PMTCT) and how counseling has helped in preventing infants from getting infected. Finally, the study also aims at understanding additional services clients want from the counseling and information centers.

One country in sub-Saharan Africa where the HIV/AIDS epidemic has been especially prevalent is Botswana, where $21.9 \%$ of adults ages 15-49 were infected with HIV in 2013 [2]. Although career guidance counseling existed in Botswana as early as 1963, it was not until the 1990's that counseling centers with a focus on mental health began to emerge [15]. These counseling centers provide services directly related to HIV/AIDS concerns, such as pre-ARV therapy, as a response to the epidemic. Counseling centers are located in hospitals, counseling clinics, and non-governmental organizations.

Stockton and his colleagues explored the experiences of 181 HIV/AIDS counselors in Botswana, through the use of both quantitative and qualitative data $[5,16]$. The majority of counselors were lay counselors (i.e., persons with two years or less of training) or other allied health professionals such as nurses and doctors [5]. This data indicated that the majority of these counselors were very satisfied with their training and perceived themselves to be effective in working with clients [16]. That being said, a large majority of counselors reported 8-weeks or less of formal training and field experience and no on-the-job supervision during an average month [16]. Both quantitative and qualitative data yielded results that participants desired much more education and training in order to be more effective counselors. In addition to expressing a desire for greater training, counselors expressed frustration for the often unclear and temporary role of lay counselors. They provided suggestions for systemic changes such as greater dissemination and coordination of services within and between hospitals and other treatment providers [5]. Although the work is stressful and can lead to burnout, the majority of counselors found their work satisfying and gratifying $[5,16]$. The data these counselors provided served as a sparkplug to improve the training and work conditions of counselors in Botswana.

With improvements implemented in the training of counselors providing services to HIV/AIDS clients in Botswana, Stockton, et al. [4] sought to obtain quantitative data on the benefits HIV/AIDS clients gain from these services in addition to qualitative data. Their study included 319 adult HIV/AIDS clients who had received counseling services in Botswana.

Among the numerous benefits observed, the authors noted that clients were positive about the benefits they received from counseling (e.g., education on practicing safe sex, the importance of taking medication regularly), the majority of participants perceived a positive relationship with their counselor, indicated a high level of satisfaction with educational materials they had received, had positive perceptions about the goal setting process in counseling, often felt that they could depend on family and friends for help when needed, and a majority of participants had positive feelings toward their present quality of life. One particularly important finding was that participants felt there was a social stigma with being HIV-positive and that others treated them differently when they knew they were HIV-positive. Although these data were helpful to elicit responses to specific questions 
and quantify clients' experiences, richer data may allow for a more in-depth understanding of the experiences clients have had while receiving counseling services in Botswana.

The current study sought to build upon the quantitative research by Stockton, et al. [4] by exploring HIV/AIDS clients' perceptions of counseling services in Botswana through the use of qualitative data. As such, the central research question was: what are patients' (clients') perceptions of HIV/AIDS counseling services in Botswana? In order to explore this central question, participants were asked to answer five open-ended questions with sub-questions in surveys assessing their experiences with counseling services. These five questions addressed: (1) topics typically addressed during counseling sessions (2) what impact being HIV-positive had on clients (3) experiences related to counseling while being pregnant with HIV/AIDS (4) the effects of disclosing HIV status to others and (5) how counseling has been most valuable to clients and their suggested improvements.

\section{Method}

\section{Participants}

Participants for the study were 328 adult clients (i.e., 18 years-old or older) who were HIV positive and receiving supportive counseling during the study. Clients were sequentially offered the opportunity to participate in the study and those who volunteered were added as participants.

Data collection began in April 2013. After the data was collected, it was found that there were some data integrity issues (it was discovered that the study protocol was not properly followed in two regions) and contaminated data had to be discarded in those regions necessitating re-submission to IRB to collect additional data. Permission was obtained and additional data was collected from new volunteers in March and April 2014.

On average, participants had known about their HIV status for several years $(\mathrm{M}=6.92, \mathrm{SD}=3.61)$. In the sample, $57.32 \%$ of participants identified as female $(n=188), 39.94 \%$ identified as male $(n=131)$, and $2.74 \%$ did not indicate their gender $(n=9)$. (Refer to Table 1 for further details on demographics.)

\begin{tabular}{|c|c|c|c|c|c|c|c|}
\hline $\begin{array}{c}\mathrm{N}=328(\mathrm{M}= \\
131 \\
\mathrm{~F}=188)\end{array}$ & $\begin{array}{c}18-25 \text { years } \\
14\end{array}$ & $\begin{array}{c}26-35 \text { years } \\
66\end{array}$ & $\begin{array}{c}36-45 \text { years } \\
111\end{array}$ & $\begin{array}{c}46-55 \text { years } \\
105\end{array}$ & $\begin{array}{c}56-65 \text { years } \\
27\end{array}$ & $\begin{array}{c}>66 \text { years } \\
4\end{array}$ & \\
\hline $\begin{array}{l}\text { Educational } \\
\text { Qualification }\end{array}$ & $\begin{array}{c}\text { No formal } \\
\text { Education } \\
137\end{array}$ & $\begin{array}{c}\text { Junior Secondary } \\
101\end{array}$ & $\begin{array}{l}\text { Senior Secondary } \\
53\end{array}$ & $\begin{array}{c}\text { Certificate } \\
14\end{array}$ & $\begin{array}{c}\text { Diploma } \\
14\end{array}$ & $\begin{array}{c}\text { Bachelor's } \\
\text { Degree } \\
5\end{array}$ & $\begin{array}{c}\text { Master's } \\
\text { Degree } \\
1\end{array}$ \\
\hline $\begin{array}{l}\text { Geographic } \\
\text { Location }\end{array}$ & Urban 82 & & Peri-Urban 112 & & Rural 130 & & \\
\hline $\begin{array}{l}\text { Employment } \\
\text { Status }\end{array}$ & \multicolumn{2}{|c|}{ Unemployed } & \multicolumn{2}{|c|}{ Working Part-Time } & \multicolumn{2}{|c|}{ Working Full-time } & $\begin{array}{c}\text { Self Employed } \\
66(20.12 \%)\end{array}$ \\
\hline
\end{tabular}

Table 1: Demographics of the participants

\section{Instrument}

A survey instrument was created and pilot tested for this study. After consulting with Botswana colleagues and officials, the survey was written in English as it is one of two national languages in Botswana and English is also taught in schools in Botswana. The responses obtained from the surveys were broken into units and those units were categorized in themes. The authors followed the recommendations of Braun and Clarke 2006 [17] relating to thematic analysis. The relative independence of the themes is established by the judges' ability to reliably categorize units into the various themes [17]. The survey contained five open-ended questions that were used to generate qualitative data. The questions used were as follows:

1. What aspects of your life does your typical counseling session focus on?

2. What impact has being HIV positive had on you?

3. If you are or have been pregnant since you have been HIV positive, please discuss your experiences with regards to your pregnancy and HIV/AIDS supportive counseling that you have received.

4. Have you disclosed your HIV status to significant people in your life? If so, please write about the experience and/or consequences of disclosing your status to them. If not, please explain what has kept you from doing so.

5. In what ways has counseling been most valuable you, and what additional help are you most in need from your counselor?

\section{Procedure}

This study received approval from the Ministry of Health in Botswana. Population centers in Botswana chosen for this study were: Gaborone, Francistown, Masunga, Tutume, Mann, Mahalapye, Palapye, Selibe-Phikwe, Molepolole, Loabtse, and Kanye. 
After randomly determining the 25 hospitals/clinics under study, volunteers who met the study's inclusion criteria were then recruited from these sites. Employees from the Institute for Development Management (the major institution in Botswana that trains HIV/AIDS paraprofessional counselors), then went to each site and explained the research study and consent procedures to potential participants. Individuals who wished to participate in the study were given a consent form to sign and complete and then given a survey to complete.

Ultimately, 329 participants signed the consent form and completed the survey, but one participant's data was excluded due to completing less than half of the survey, leaving 328 usable surveys. As noted earlier during initial data collection, it was found that the study protocol was not followed in two of the study's population centers, therefore this data was excluded from study, and data was recollected at sites in these areas, following the study's protocol.

\section{Data Analysis}

In order to analyze the qualitative responses, thematic analysis was utilized [18]. Data was approached with a realist epistemology in that the responses of the participants were taken at face value in order to understand their experiences with counseling [17]. In order to carry out this epistemology, researchers used deductive analysis by breaking down responses to survey questions into separate units based on semantic themes. Semantic themes are explicit themes found at the surface level within the data that explicitly address survey questions being asked [17]. Coinciding with recommendations by Braun and Clarke [17] semantic themes were determined once all researchers on the research team had a chance to familiarize themselves with the data. Once familiar with the data, responses were broken down (i.e., coded) into units that shared similarities with one another. After units had been determined, the research team met to define themes that would be used to categorize units the research team had extracted from the data.

Finally, once themes had been identified, each member of the research team paired up with a partner on the research team and each pair was assigned a survey question to analyze. Pairs then split up and examined all response units to the question they had been assigned. Researchers categorized units into the semantic themes that had been identified during the research team meeting. After individuals categorized units, they reconvened with their partner and compared their categorization of units to their partner's categorization. If both members of the pair had separately categorized a unit into the same theme, they were determined to be in "agreement." If partners did not categorize a unit into the same theme initially, but were able to agree on categorization after reconvening, they were determined to be in "consensus". If partners were not able to come to a consensus on how a unit should be categorized, it was considered that partners had reached a point of "no consensus," for that unit. If there was no consensus reached amongst pairs, then that unit was brought back to the full team, and if the team could not reach a consensus, the unit was categorized as having no consensus. For a summary of the agreement on themes between raters, Tables 2-6.

\begin{tabular}{|c|c|c|c|c|c|}
\hline Theme & No. of Units & No. of Participants & \multicolumn{3}{|c|}{ Agreement } \\
\hline Total & 853 & 242 & \% Agreement & $\%$ Consensus & $\%$ No Consensus \\
\hline Acceptance of Status/Self & 26 & 24 & 96.25 & 3.05 & 0.70 \\
\hline Education & 723 & 233 & & & \\
\hline General Education & 158 & 122 & & & \\
\hline Alcohol and Drug Issues & 76 & 74 & & & \\
\hline Available Resources & 10 & 9 & & & \\
\hline Medication Adherence & 207 & 162 & & & \\
\hline Nutritional Needs & 66 & 65 & & & \\
\hline Safe Sex Practices & 272 & 178 & & & \\
\hline $\begin{array}{l}\text { Encouragement of Healthy } \\
\text { Behaviors for Others }\end{array}$ & 1 & 1 & & & \\
\hline How to improve counseling & 1 & 1 & & & \\
\hline Instillation of Hope & 15 & 12 & & & \\
\hline No Theme & 12 & 12 & & & \\
\hline
\end{tabular}

Note: Number of units in the total row reflects the total number of units generated by participants. Number of units in each theme's row reflects only those units which raters reached agreement or consensus on in their coding.

Table 2: What aspects of your life does your typical counseling session focus on?

\section{Results}

Although thematic analysis generated many themes to each question (Table 2-6 for a complete list of themes), this section will only include the top three themes found for each question. Additionally, all quotes have been corrected for spelling and/or grammar error. 


\section{Focus of Counseling Sessions}

For the purposes of this report, $u$ will refer to the number of units in a given theme or sub theme and $\mathrm{n}$ will refer to the number of participants that generated those units.

The first open-ended question of the survey used in this study asked participants, "What aspects of your life does your typical counseling session focus on?" A total of 242 participants responded to this question; responses were then broken down into 853 units of responses, which were then coded according to common themes. Of the 853 units of responses, team members were in agreement on the coding of 821 units, 26 units were coded by consensus between team members, and consensus could not be reached on 6 units. On coding participants' responses, several major themes emerged. By far the number one major theme that was produced in response to this question was education, which was exemplified in 723 units generated by 233 participants. Responses in this domain were then broken into six sub-themes: safe sex practices $(u=272, n=178)$, medication adherence $(u=207, n=162)$, alcohol and drug issues ( $u=76, n=64)$, nutritional needs $(u=66, n=65)$, available resources $(u=10, n=9)$, and the remaining 158 units $(n=122)$ that fell under the theme of education but could not be categorized into one of the five previous sub- themes were categorized as general education (see Table 2 ).

The first education sub-theme was safe sex practices. Safe sex practices referred to the use of condoms, reducing the number of sexual partners, and how to handle situations with sexual partners who did not want to practice safe sex. Additionally, general education about how HIV/AIDS is contracted and spread and general prevention information was also grouped into this domain. Answers like "safe sex practices," "stick to one partner," "always use a condom," and "A-abstinence, B- be faithful, C- condomize" were common throughout.

The second sub-theme was medication adherence. "Always take medication on time," "attend doctor's appointments regularly," "avoid traditional herbs/medicines," "do not share medication," and "these medications will be taken for the rest of my life" were common responses detailing the compliance of medication adherence and ongoing medical treatment. The third sub-theme alcohol and drug issues -noted that issues related to alcohol, drug, and tobacco use were often discussed in counseling sessions. Typical responses included "avoid drugs," "no drinking alcohol," and "no tobacco use during this treatment." The forth sub-theme was nutritional needs, which referred to units of responses where participants acknowledge that counseling sessions relayed the importance of diet and eating healthy foods.

The smallest sub-theme identified under education was labeled available resources. This sub-theme referred to the information participants received regarding resources available to them during counseling sessions. Examples of the available resources cited by participants were "HIV/AIDS counseling," and "introduction to PMTCT program." Typical responses in the general education sub-theme were, "behavior change," "exercise," "HIV/AIDS issues," "secondary infection," and "health issues." Perhaps this sub theme is best described as a "catch-all" for general, if not vague, responses related to educational topics discussed in counseling.

The second greatest distinct, major theme found by the research team was acceptance of status/self $(u=26, n=24)$. Participants primarily identified "self-acceptance," acceptance of status and medical needs, and the importance of "coping" and "acceptance" as topics discussed with their counselor. To supplement the theme of acceptance of status/self, 14 respondents' answers were coded under the third major, distinct theme of responses to this question - instillation of hope $(u=15)$. Instillation of hope included statements about "living positively" and embracing the belief that, "this is not the end of life."

\section{The Negative Impact of Being HIV-positive in Botswana}

The second open-ended question of the survey asked participants, "What impact has being HIV-positive had on you?" This question was designed to elicit personal experiences of those living with a positive HIV status in Botswana. The purpose of identifying these experiences is to educate counselors with hopes it will improve practice and also to demonstrate the need for counseling within the population. A total of 666 units of responses, across 296 participants, were identified for this question about the impact of being HIV-positive. Of the 666, total units, 611 were coded in agreement, 54 reached consensus, and only 1 unit was not able to reach agreement or consensus. A number of themes that emerged from this question were related to the negative impact of being HIV positive. The top 3 themes that this data produced were: discrimination encountered $(u=102, n=73)$, psychological distress $(u=$ 91, $n=64)$, and fear $(u=66, n=54$; Table 3). The top theme coded for the impact of being HIV-positive related to participants' experiences of encountering discrimination. Participants encountered discrimination from a broad range of individuals in their lives including family members, employers, partners, friends and society in general. Discrimination included being a subject of gossip, receiving dirty looks, individuals being persecuted for their status and having assumptions made about how they became HIV positive. Furthermore, many responses included phrases that indicated important people in participants' lives had left them and would no longer speak to those with a positive status, such as, "My girlfriend dumped me," "... my family stopped giving me the love they used to give me," and, "All my friends walked away from me when they knew I was positive."

The second-most frequently coded theme to question 2 was psychological distress. Respondents described feeling guilty, stressed about their new status, depressed, and alone. Examples of units that were coded under this theme were statements such as, “. . I feel like there is no hope in life," Being HIV positive has shattered a lot of dreams and plans I had for my life," and "When I came to the reality that I was HIV positive I could not accept myself." 


\begin{tabular}{|c|c|c|c|c|c|}
\hline Theme & No. of Units & No. of Participants & \multicolumn{3}{|c|}{ Agreement } \\
\hline Total & 666 & 296 & \% Agreement & \% Consensus & $\%$ No Consensus \\
\hline Acceptance of Status/Self & 44 & 43 & 91.74 & 8.11 & 0.15 \\
\hline $\begin{array}{l}\text { Counseling and Benefits of } \\
\text { Counseling }\end{array}$ & 13 & 11 & & & \\
\hline Career/Job Problems & 40 & 31 & & & \\
\hline Discrimination Encountered & 102 & 73 & & & \\
\hline Education & 3 & 3 & & & \\
\hline Fear & 66 & 54 & & & \\
\hline Hiding HIV/AIDS Status & 22 & 17 & & & \\
\hline Instillation of Hope & 4 & 4 & & & \\
\hline Interpersonal Impacts & 45 & 41 & & & \\
\hline Job Problems & 23 & 19 & & & \\
\hline Living in the Present & 2 & 2 & & & \\
\hline Miscellaneous & 26 & 22 & & & \\
\hline $\begin{array}{l}\text { Negative Impact of Being HIV } \\
\text { Positive }\end{array}$ & 8 & 6 & & & \\
\hline $\begin{array}{l}\text { Negative physical/behavioral } \\
\text { disturbance }\end{array}$ & 44 & 38 & & & \\
\hline No Theme & 32 & 32 & & & \\
\hline No impact & 14 & 14 & & & \\
\hline Physical Impact & 14 & 14 & & & \\
\hline Psychological Distress & 91 & 64 & & & \\
\hline Safe Sex Practice & 4 & 3 & & & \\
\hline Support & 20 & 19 & & & \\
\hline
\end{tabular}

The third theme, fear, was a theme that respondents wrote about extensively. The respondents identified experiencing fear in many ways such as being afraid of death, isolation, discrimination, and the future. Respondents feared discrimination from their family, employers, partners, and social groups, and rightfully so considering the top-coded theme for this question. Fear of the future was categorized by fear for their current or potential children (mainly if they will be infected or experience a negative impact from their parent's status), fear of the future of a relationship with a significant other or partner, fear of the well-being of one's family, and fear of securing/maintaining a job in the future.

Examples of units that were coded under the theme of fear are, "Being HIV positive children," "Big impact because I fear my friend would isolate me if they know I am HIV positive," and, "The first thing that came to mind was death, I was, and still am, afraid to die."

\section{Experiences Regarding Pregnancy and HIV/AIDS in Counseling}

Question three of the survey asked: "If you are or have been pregnant since you have been HIV positive, please discuss your experiences with regards to your pregnancy and HIV/AIDS supportive counseling that you have received." There were 215 units of responses generated by 67 participants who responded to this question. Popular themes that were reported were Preventing Mother to Child Transmission (PMTCT) helping to have HIV-negative children $(u=92, n=43)$, education $(u=19, n=17)$, and counseling and the benefits of counseling $(u=11, n=10$; Table 4). Of the 215 total units coded, 200 were in agreement and 15 reached consensus, leaving no units in a state of no consensus.

The most common response to this question concerned the result of enrollment in the PMTCT program (i.e., PMTCT helping to have HIV-negative children), which aims to helps educate mothers concerning medicine and behaviors that can help prevent the passage of HIV/AIDS to their children. The majority of participants appeared quite pleased with the results of enlisting in this program, which is evident in the responses such as, "Received counseling and was in PMTCT program. I now have $3 \mathrm{HIV}$-negative children," "Was in PMTCT and my child is HIV negative," and "I was enrolled for PMTCT and due to counseling I had a normal pregnancy. I gave birth to a healthy baby."

The second most common theme coded in response to question 3 was education. Similar to question I, education indicated knowledge gained from the process of counseling itself. The theme of education mostly referred to clients' discovery of the PMTCT program as a result of counseling, but also included things such as dietary suggestions and how often to visit a doctor. Two examples of such responses include, "I enrolled for PMTCT, I was told what will happen to my baby (that he will be HIV-negative) now that I have enrolled for = PMTCT", and "The counselor told me to join PMTCT and she told me that this program would prevent the virus from passing to the newborn." 


\begin{tabular}{|c|c|c|c|c|c|}
\hline Theme & No. of Units & No. of Participants & \multicolumn{3}{|c|}{ Agreement } \\
\hline Total & 215 & 67 & \% Agreement & $\%$ Consensus & $\%$ No Consensus \\
\hline Acceptance & 2 & 2 & 93.02 & 6.98 & 0.00 \\
\hline Counseling and Benefits of Counseling & 11 & 10 & & & \\
\hline Education & 19 & 17 & & & \\
\hline Fear of Future & 5 & 3 & & & \\
\hline Instillation of Hope & 5 & 5 & & & \\
\hline Medication adherence & 2 & 2 & & & \\
\hline Miscellaneous & 20 & 13 & & & \\
\hline $\begin{array}{l}\text { Negative Physical/Behavioral Distur- } \\
\text { bance }\end{array}$ & 1 & 1 & & & \\
\hline No theme & 44 & 29 & & & \\
\hline $\begin{array}{l}\text { PMTCT Helping to Have HIV Negative } \\
\text { Children }\end{array}$ & 92 & 43 & & & \\
\hline Psychological Distress & 9 & 6 & & & \\
\hline Support & 5 & 5 & & & \\
\hline
\end{tabular}

Table 4: If you are or have been pregnant since you have been HIV positive, please discuss your experiences with regards to your pregnancy and HIV/AIDS supportive counseling that you have received

The third most popular theme was counseling and the benefits of counseling. Units coded under this theme referred to doctors' referrals to counseling and what participants viewed as the positive outcomes they gained from counseling while pregnant. Examples included statements such as, "... supportive counseling made my pregnancy seem normal, I had no complications whatsoever," and, "I was a bit crushed and hurt, but I was given counseling and I felt better."

\section{The Effects of Disclosing HIV-Positive Status}

A significant aim of this study was to gain an increased awareness of participants' interpersonal experiences related to a positive HIV/AIDS status. One survey question was posed to participants in order to gain insight on patterns of disclosing HIV/AIDS status, as well as the benefits and consequences of this disclosure. This question was, "Have you disclosed your HIV status to significant people in your life? If so, please write about the experience and/or consequences of disclosing your status to them. If not, please explain what has kept you from doing so." A total of 308 participants responded to this survey question, which yielded a total of 1076 units of responses. Out of the 1076 total units, coders were in agreement on 1019 of them, reached consensus on 53, and only 4 units were left at no consensus by the research team (Table 5).

\begin{tabular}{|c|c|c|c|c|c|}
\hline Theme & No. of Units & No. of Participants & \multicolumn{3}{|c|}{ Agreement } \\
\hline Total & 1076 & 308 & \% Agreement & $\%$ Consensus & \% No Consensus \\
\hline Acceptance of Status/Self & 36 & 36 & 94.34 & 5.55 & 0.37 \\
\hline Career/Job Problem & 2 & 2 & & & \\
\hline Disclosed & 364 & 261 & & & \\
\hline Discrimination Encountered & 55 & 41 & & & \\
\hline Educating Others & 4 & 4 & & & \\
\hline Education & 4 & 3 & & & \\
\hline Encouragement of Healthy Behaviors for Others & 5 & 5 & & & \\
\hline Fear & 95 & 71 & & & \\
\hline Hiding HIV/AIDS Status & 17 & 12 & & & \\
\hline Instillation of Hope & 3 & 3 & & & \\
\hline Interpersonal Impact & 39 & 32 & & & \\
\hline Miscellaneous & 41 & 31 & & & \\
\hline Negative Physical/Behavioral Disturbance & 2 & 2 & & & \\
\hline No Impact & 1 & 1 & & & \\
\hline No Theme & 41 & 36 & & & \\
\hline PMTCT Helping to HIV Negative Children & 1 & 1 & & & \\
\hline Psychological Distress & 4 & 4 & & & \\
\hline Support & 323 & 217 & & & \\
\hline
\end{tabular}

Table 5: Have you disclosed your HIV status to significant people in your life? If so, please write about the experience and/or consequences of disclosing your status to them. If not, please explain what has kept you from doing so 
The responses indicated that the majority of survey respondents tended to disclose their status, with a total of 364 units from 261 survey respondents indicating disclosure to at least one person in their lives. The overwhelming feedback was that the majority of participants received support after disclosing their status to significant others $(u=323, n=217)$. Many participants either did not disclose their status or were hesitant to disclose their status due to fear $(u=95, n=71)$ and unfortunately, the third most frequent theme as an effect of disclosure was discrimination encountered $(u=55, n=41)$. Although many participants received negative responses after disclosing their status to significant others, a large portion of survey respondents reported more positive responses. These positive responses were coded under the theme of support. Units that indicated participants received support were statements such as, "all people who now are giving me love, support, and care at all times," "... the experience is that they always encourage me to talk to them when I am having any problems," and, "... the experience is that they are always there for me in the ups and downs."

Whether or not participants reported disclosing their status, a noteworthy emergent theme was a fear related to possible disclosure. For example, participants indicated a fear that others would think badly of their moral character or that unfavorable rumors may be spread about them. Others indicated a fear of more overt, societal discrimination, specifically from employers. One participant stated, "At work, I do think if I tell them they will terminate my contract." Another participant stated, "They might think I am too weak to deliver quality services." Other survey respondents indicated a theme of fear of isolation and rejection, "It took me some months to disclose my status to the people in my life because I feared they would isolate me." Although a large number of survey respondents indicated having fears related to disclosing their status, the numbers suggest that these fears often times did not keep individuals from disclosing their status. Many fears related to discrimination and isolation was realized after survey respondents disclosed their status to others as indicated by the third-most frequent theme: discrimination encountered. Similar to the responses coded in question 2, respondents indicated that they encountered discrimination after disclosure, such as a decreased status in the family, partners leaving as a result of the disclosure, and even violence and abuse from partners. Examples of such statements included, "There is discrimination especially from my family. My mother always tells my children that one day I will be dead because I am HIV positive," "I also disclosed my status to the man I was dating when I tested positive and he left me," and, “... he beat me up."

\section{How Counseling Was Most Valuable}

In order to understand what clients perceived to be the positive aspects of their experience as well as how services could be improved, clients were asked: "In what ways has counseling been most valuable to you, and what additional help are you most in need from your counselor?" Participants provided a plethora of responses $(u=725, n=242)$ that were collapsed into themes for both portions of the question. There were 663 units that coders were in agreement on, 61 that coders reached consensus on, and 1 unit that the research team was not able to reach consensus on (Table 6).

\begin{tabular}{|c|c|c|c|c|c|}
\hline Theme & No. of Units & No. of Participants & \multicolumn{3}{|c|}{ Agreement } \\
\hline Total & 725 & 242 & \% Agreement & $\%$ Consensus & $\%$ No Consensus \\
\hline Acceptance of Status/Self & 138 & 112 & 91.45 & 8.41 & 0.14 \\
\hline Discrimination Encountered & 1 & 1 & & & \\
\hline Education & 271 & 151 & & & \\
\hline $\begin{array}{c}\text { Encouragement of Healthy Behaviors for } \\
\text { Others }\end{array}$ & 31 & 27 & & & \\
\hline Instillation of Hope & 86 & 78 & & & \\
\hline Interpersonal Benefits & 9 & 8 & & & \\
\hline Intrapersonal Benefits & 6 & 6 & & & \\
\hline Miscellaneous & 58 & 49 & & & \\
\hline Not Helpful & 8 & 5 & & & \\
\hline More Counselor Training & 4 & 4 & & & \\
\hline More Information About Resources/Programs & 6 & 5 & & & \\
\hline Need for Home Visit & 1 & 1 & & & \\
\hline Negative Physical/Behavioral Disturbance & 2 & 2 & & & \\
\hline No Theme & 24 & 19 & & & \\
\hline $\begin{array}{l}\text { PMTCT Helping to Have HIV- Negative } \\
\text { Children }\end{array}$ & 7 & 7 & & & \\
\hline Positive Aspects Despite Being HIV Positive & 1 & 1 & & & \\
\hline Positive Aspects/Feedback & 2 & 2 & & & \\
\hline Psychological Distress & 4 & 4 & & & \\
\hline Request for More Resources & 31 & 30 & & & \\
\hline Request for More Sessions & 34 & 32 & & & \\
\hline
\end{tabular}

Table 6: In what ways has counseling been most valuable to you, and what additional help are you most in need from your counselor? 
Journal of Immunology and Infectious Diseases

In answer to the question, "In what ways has counseling been most valuable to you," the three most common themes that occurred were: education $(u=271, n=151)$, acceptance of status/self $(u=138, n=112)$, and instillation of hope $(u=86, n=78)$. Responses that fell under the theme of education were similar to responses observed in question 1 and included units such as, "the importance of medication adherence," "practice safe sex (consistence condom use)," "to protect my child from HIV/AIDS," and, "eat fruit and vegetables ... and not drink alcohol." Units that were coded as acceptance of status/self-included, "I managed to accept my status," "feel so free and open to talk about HIV/AIDS issues no matter where I am," "don't blame myself," and "helped me to boost my confidence back within the community." Instillation of hope included statements such as, "this is not the end of life," and "to know that HIV is just a virus not a disease and I can still live longer than I thought."

\section{Suggested Improvements}

When responses to the second portion of the fifth question (i.e., "what additional help are you most in need of from you counselor?") were examined, the top three themes that were found were requests for more sessions $(u=34, n=32)$, requests for more resources $(u=31, \underline{n}=30)$, and more information about resources/programs $(u=6, n=5)$. The first major theme, request for more sessions, included responses such as, "in addition, I would like our counselors to counsel us as a group just for a day so we can hear how others are living since they are positive and this will help us to teach each other how to cope with this status," "In addition I want them to counsel us every check-up because mostly they give us counseling only when you start medication and only when you ask for it" and "Counsellors should help us with counseling every time we come and see the doctor to help those who are dying inside like me." Units that were categorized under the second major theme, request for more resources, alluded to clients' desire to have basic needs met through the counseling services provided to them. One respondent encapsulated many participants' sentiments in this theme with one sentence, "Counsellors should provide orphans with allowance, transport, food baskets and shelter." An example statement from the third major theme, more information about resources/programs, was, "At the moment I feel I am not in need of any help from my counselor but I would appreciate anything that is new that I should know about HIV/AIDS."

\section{Discussion}

This qualitative study focuses on client experiences regarding HIV/AIDS counseling in Botswana. It seeks to understand the psychological experiences of the clients. It is not only important to understand the progress made by providing counseling sessions but to also understand how the counseling sessions can be improved to ensure psychological well-being of people with a life threatening virus/disease such as HIV/AIDS.

It seems clear that the primary emphasis in counseling sessions, for clients in this study, has been on the psycho educational aspects of dealing with HIV/AIDS. “The current study found that counseling sessions focused primarily on education about HIV/ AIDS, including safe sex practices, medical compliance, alcohol and drug issues, nutritional needs, general education about HIV/ AIDS and its contraction, spread and prevention, and information regarding available resources". Additionally, counseling sessions focused on instillation of hope, self-acceptance and coping with the social stigma associated with the disease. According to client reports, counseling appeared to have positively impacted clients' physical and mental health. Similar results regarding positive effects of counseling have also been found in other studies involving individuals with HIV/AIDS [19,20].

The current study is consistent with previous findings that HIV/AIDS negatively impacts physical and mental health of clients inducing behavioral problems, fear (e.g. of death, job complications, potential discrimination) depression, loss of appetite, loss of sex drive and possibly experiencing prejudiced attitudes from others [21-23]. This study found that the clients experienced major negative impacts due to encountered discrimination followed by psychological distress due to guilt and loneliness. Additionally, clients were surrounded by fearful feelings of death, discrimination and isolation, social stigmatization, despair over uncertain career prospects, lifelong medication, restricted sex life, and fear for current and potential children. The current study also found that clients were fearful of disclosing their status due to stigma attached to the condition. Lastly, findings of this study suggest that clients encounter several negative physical and behavioral disturbances which include a decreased sex drive, depression, increase in substance use to forget about their status, and even suicidal thoughts. As indicated earlier, much of the focus of counseling sessions, as reported by clients, had been on psycho education. However, the clients' comments concerning the impact of HIV/AIDS in their lives indicate a need to also emphasize the social/emotional aspects of client needs for those with HIV/AIDS in Botswana.

In Sub Saharan Africa half of the HIV positive population is women. This puts unborn children at risk of getting infected. The comments of participants who were mothers demonstrated that counseling played a crucial role in saving their babies from becoming infected with HIV; this major impact was brought about by women emailing in the Prevention of Mother to Child Transmission (PMTCT) program. By educating women about necessary precautions to take before, during, and after pregnancy, and providing necessary support and resources, mother to child transmission of HIV is lowered. The current study has found that the majority of Botswana mothers reported having benefited from, and were pleased, by the PMTCT services provided by the counseling centers. The risk of mother to child transmission of HIV is reduced considerably if women know their status and are thus able to make more informed choices about their reproductive lives, which resonates with past research [e.g., 24]. Given the positive response of mothers in the study to the PMTCT program, it seems imperative to emphasize its importance when counseling pregnant mothers and female clients of child-bearing age. 
Another significant finding of this study is participants' experiences in disclosing their HIV status. Although a majority of participants disclosed their status to at least one other person, many did not report disclosing to anyone. As found in this study, participants feared being judged and questioned on their moral character and feared losing their career because of disclosure. Gilbert and Walker (2010) report that the main reason behind lack of disclosure to family, employers and intimate/ sexual partners is fear of stigmatization [25].

Similarly, an important theme that emerged in the current study, irrespective of disclosing one's HIV/AIDS status or not, was fear. Participants feared discrimination (especially at work), social isolation and rejection, and lowered status in the family. The current study found that unfortunately many of these fears were realized after disclosure, as respondents encountered discrimination, abandonment, decreased family status, and even abuse from relationship partners.

Although disclosure comes at a cost, it was found in the current study and other studies as well, that disclosing one's status to family members and friends can relieve the stress of concealing one's HIV-status and create access to social support [26], which may buffer emotional distress in people living with HIV-AIDS [27]. Even though the personal costs may be high, it is emotionally beneficial for people to disclose their HIV status and, according to Paxton (2002) [28], disclosure can be the most liberating course of action. This was found to be true for many of the participants in the current study. The majority of participants reported positive responses and support from significant others after disclosure. Cohen and Wills (1985) contend that support from significant others buffers the impact of a wide variety of stressful life events including chronic illness. Given the support for the positive results of disclosing HIV status to significant others as well as potential negative consequences, it seems important to emphasize in counselor training the risks and benefits associated with the decision to either disclose or not disclose one's HIV/AIDS status in Botswana. Counselors working with this population need to be able to appropriately discuss with clients their decision whether or not to disclose their HIV/AIDS status.

Finally, in order to understand clients' perceptions of the counseling received and to gain feedback for further improvements, they were asked about positive aspects of their counseling experience and suggested improvements. Results of the current study indicated that counseling has been most beneficial in providing education about HIV/AIDS, medical adherence, safe sex practices, acceptance of self, and instillation of hope for clients. Clients reported being quite satisfied by the counseling provided to them. These findings are in congruence with the findings of a quantitative study conducted by Stockton, Paul, Morran, and Mokalake [4], which obtained data on the benefits HIV/AIDS clients gain from counseling services in Botswana. In the current study, there were three main themes that emerged from the suggestions made by participants to improve their counseling experience. First, to increase the number of counseling sessions and to provide sessions after every check-up as counseling helps in coping with the stress associated with the HIV status. The second suggestion was a request for more resources from the counseling centers. In a country with declining job prospects many participants hoped for more help from the counseling centers. Lastly, clients also expected to regularly receive any new and relevant information regarding HIV/AIDS.

As with all studies, this one has limitations. Participants were restricted to one country (i.e., Botswana) and were volunteers; therefore findings may not generalize to other countries with their unique cultures. Additionally, although care was taken to properly identify and code the themes that emerged from the study, they are of necessity subjective and open to different interpretations. Despite these limitations, the information gained does have significant value for HIV/AIDS counseling in Botswana and potentially in other countries.

\section{Recommendations and Conclusion}

This study was an in depth qualitative research effort based on feedback and comments from HIV positive clients regarding their counseling. It is important to understand that, in addition to the powerful and lifesaving effects of the medicine and the research that goes into developing biological treatment, the social emotional components are also important and have not been studied as extensively as other areas. Tentative recommendations include consideration regarding expanding the focus of counseling beyond psychoeducational topics to include social/emotional areas faced by clients, increasing the number and length of counseling sessions, emphasizing the value of PMTCT programs for female clients who are pregnant or of child-bearing age, specifically engaging clients in a discussion of the pros and cons of disclosure of their HIV status to appropriate others and putting into place a mechanism for systematically informing clients of new information or developments in HIV/AIDS treatment. Future initiatives could be taken to ensure better services to the patients.

\section{Author Note}

Special thanks to members of the International Counseling, Advocacy, Research, \& Education (I-CARE) team at Indiana University's School of Education, whose contributions were instrumental to the current research study.

\section{References}

1. UNAIDS (2015) AIDS by the numbers 2015. The Joint United Nations Pogramme on HIV/AIDS (UNAIDS).

2. World Health Organization (2015) Botswana statistics summary. 
3. UNAIDS (2015). Fact sheet 2015. The Joint United Nations Pogramme on HIV/AIDS (UNAIDS).

4. Stockton R, Paul T, Morran DK, Mokalake E (2016) Survey of HIV/AIDS clients in botswana: Reactions to supportive counseling. Inte J Adv Counsel 38: 249-68.

5. Stockton R, Paul T, Voils-Levanda A, Robbins M, Li P, et al. (2015) Counselors' perceptions of HIV/AIDS counseling in Botswana: Professional identity, practice, and training issues. Int J Adv Counsel 37: 143-54.

6. Parsons JT, Rosof E, Punzlan JC, DiMaria L (2005) Integration of motivational interviewing and cognitive behavioral therapy to improve HIV medication adherence and reduce substance use among HIV-positive men and women: Results of a pilot project. AIDS Patient Care and STDs 19: 31-9.

7. Richards KAM, Marquez J (2005) Experiences of HIV/AIDS colmselors and their perceptions on the state of HIV/AIDS counseling in Zimbabwe. Int J Adv Counsel 27: 413-29.

8. Grinstead OA, Van Der Straten A (2000) The Voluntary HIV-1 Counseling and Testing Efficacy Study Group. Counsellors' perspectives on the experience of providing HIV counseling in Kenya and Tanzania: The voluntary HIV- I counseling and testing efficacy study. AIDS Care 12: 625-42.

9. Ostermann J, Reddy EA, Shorter MM, Muiruri C, Mtalo A, et al. (2011) Who tests, who doesn't, and why? Uptake of mobile HIV counseling and testing in the Kilimanjaro Region of Tanzania. PLoS One 6: e16488.

10. Van Rooyen H, McGrath N, Chirowodza A, Joseph P, Fiamma A, et al. (2013) Mobile VCT: reaching men and young people in urban and rural South African pilot studies (NIMH Project Accept, HPTN 043). AIDS Behav 17: 2946-53.

11. Mall S, Middelkoop K, Mark D, Wood R, Bekker LG (2013) Changing patterns in HIV/AIDS stigma and uptake of voluntary counselling and testing services: the results of two consecutive community surveys conducted in the Western Cape, South Africa. AIDS care 25: 194-201.

12. Schacham E, Reece M, Ongor WO, Omollo O, Monahan PO, et al. (2008) Characteristics of psychosocial support seeking during HIV-related treatment in western Kenya. AIDS Patient Care and STDs 22: 595-601.

13. Edwards SD, Edwards DJ (2009) Counseling for psychological well-being in persons living with HIV/AIDS. J Psychol in Africa 19: 561-4.

14. World Health Organization (2015) From MDGs to SDGs, WHO launches new report.

15. Stockton R, Nitza A, Buhusumane DB (2010) The development of professional counseling in Botswana. J Counsel Dev 88: 9-12.

16. Stockton R, Paul T, Morran DK, Yebei P, Chang S, et al. (2012) A survey of HIV/AIDS counselors in Botswana: Satisfaction with training and supervision, selfperceived effectiveness and reactions to counseling HIV positive clients. J HIV/AIDS Soc Servic 11: 424-46.

17. Braun V, Clarke V (2006) Using thematic analysis in psychology. Qualitative Research in Psychology 3: 77-101.

18. Fereday J, Muir-Cochrane E (2006) Demonstrating rigor using thematic analysis: A hybrid approach of inductive and deductive coding and theme development. Int J Qual Meth 5: 80-92.

19. Schmitz MF, Crystal S (2000) Social relations, coping, and psychological distress among persons with HIV/AIDS. J Appl Soc Psychol 30: 665-85.

20. Parsons JT, Rosof E, Punzalan JC, Maria LD (2005) Integration of motivational interviewing and cognitive behavioral therapy to improve HIV medication adherence and reduce substance use among HIV-positive men and women: Results of a pilot project. AIDS Patient Care STDs 19: 31-9.

21. Safren SA, Gershuny BS, Hendriksen E (2003) Symptoms of posttraumatic stress and death anxiety in persons with HN and medication adherence difficulties. AIDS Patient Care STDs 17: 657-64.

22. Herek GM, Capitanio JP (1999) AIDS stigma and sexual prejudice. American Behavioral Scientist 42: 1130-47.

23. Morin SF, Charles KA, Malyon AK (1984) The psychological impact of AIDS on gay men. Am Psychol 39: 1288-93.

24. Medley A, Garcia-Moreno C, McGill S, Maman S (2004) Rates, barriers and outcomes of HIV serostatus disclosure among women in developing countries: Implications for prevention of mother-to-child transmission programmes. Bull World Health Organ 82: 299-307.

25. Gilbert L, Walker L (2010) 'My biggest fear was that people would reject me once they knew my status. ..: Stigma as experienced by patients in an HN/AIDS clinic in Johannesburg, South Africa. Health Soc Care in the Community 18: 139-46.

26. Simoni JM, Mason HR, Marks G, Ruiz MS, Reed D, et al. (1995) Women’s self-disclosure of HIV infection: Rates, reasons, and reactions. J Consult Clin Psycho 63: 474-8,

27. Kalichman SC, DiMarco M, Austin J, Luke W, DiFonzo K (2003) Stress, social suppoti, and HIV-status disclosure to family and friends among HIV-positive men and women. J Behav Med 26: 315-32.

28. Paxton S (2002) The paradox of public HIV disclosure. AIDS Care 14: 559-67.

29. Cohen S, Wills TA (1985) Stress, social support, and the buffering hypothesis. Psychol Bull 98: 310-57.

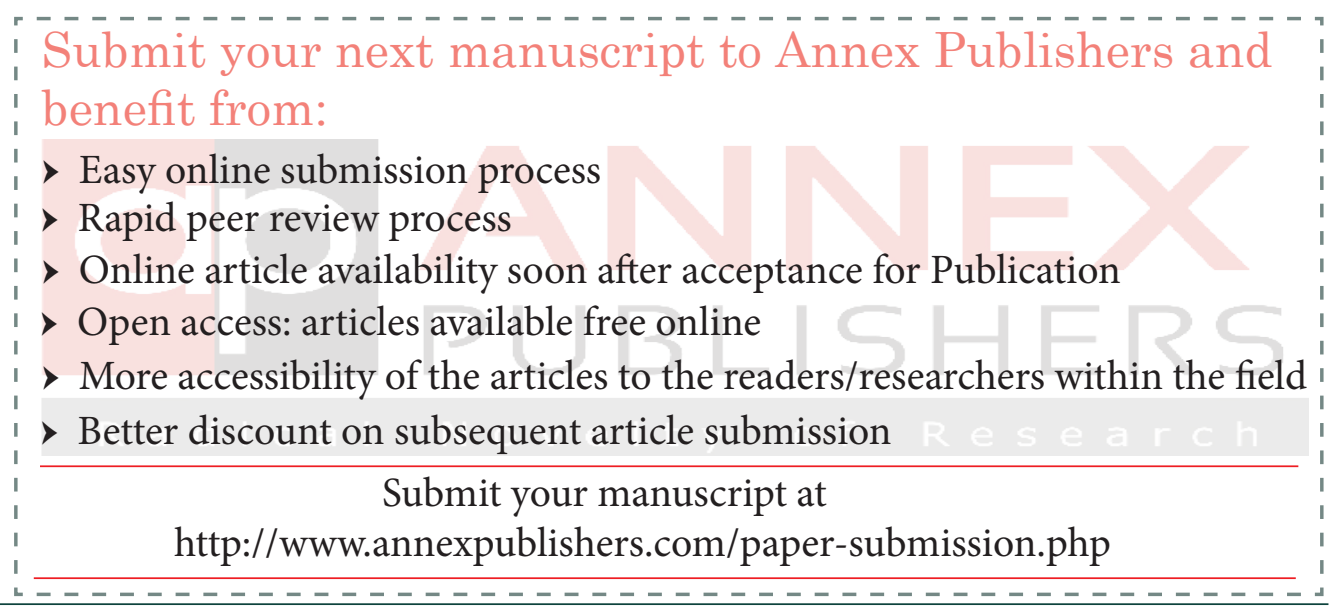

